\title{
Article \\ Chained Data Acquisition and Transmission System Protype for Cabled Seafloor Earthquake Observatory
}

\author{
Jingyang Qiao ${ }^{1,2}$, Wu Liu $^{1, * \mathbb{C}}$, Jingquan Liu ${ }^{1}$ and Jianping Zhou ${ }^{3,4}$ \\ 1 National Key Laboratory of Science and Technology on Micro/Nano Fabrication, Shanghai Jiao Tong \\ University, Shanghai 200240, China; qjy19971022@sjtu.edu.cn (J.Q.); jqliu@sjtu.edu.cn (J.L.) \\ 2 Department of Micro/Nano Electronics, School of Electronic Information and Electrical Engineering, \\ Shanghai Jiao Tong University, Shanghai 200240, China \\ 3 Key Laboratory of Submarine Geosciences, Ministry of Natural Resources, Hangzhou 310012, China; \\ zhoujp@sio.org.cn \\ 4 Second Institute of Oceanography, Ministry of Natural Resources, Hangzhou 310012, China \\ * Correspondence: liuwu@sjtu.edu.cn; Tel.: +86-021-34206400
}

check for updates

Citation: Qiao, J.; Liu, W.; Liu, J.; Zhou, J. Chained Data Acquisition and Transmission System Protype for Cabled Seafloor Earthquake Observatory. J. Mar. Sci. Eng. 2021, 9, 880. https://doi.org/10.3390/ jmse 9080880

Academic Editor: Dong-Sheng Jeng

Received: 18 July 2021

Accepted: 13 August 2021

Published: 15 August 2021

Publisher's Note: MDPI stays neutral with regard to jurisdictional claims in published maps and institutional affiliations.

Copyright: (c) 2021 by the authors. Licensee MDPI, Basel, Switzerland. This article is an open access article distributed under the terms and conditions of the Creative Commons Attribution (CC BY) license (https:// creativecommons.org/licenses/by/ $4.0 /)$.

\begin{abstract}
Seafloor observatories can provide long-term, real-time submarine monitoring data, which has great significance for the study of major scientific technology in marine science, especially in the seafloor earthquake observation. The chained submarine data sampling and transmission system is the prototype and foundation of cabled seafloor earthquake observatories. This paper designs and builds a chained data sampling and transmission system (SQSTS) based on Zynq-7000 Soc (System on chip) and clock synchronization. At the beginning, we realized high-precision submarine data (24 bit) sampling based on Zynq-7000 Soc and ADS 1256. Using the PPS (Pulse per second) signal provided by the P88 1588 PTP (Precise time protocol) clock synchronization board and the inner crystal oscillator of the Zynq-7000 Soc, the time stamp up to the microsecond level, for the seismic data sampled in each seismometer node can support subsequent inversion of seismic data. In addition, a high-speed data transmission link connecting nodes in SQSTS, which is based on the Gigabit transceiver and optical cable, has been investigated. The transmission link has been realized by using the Aurora IP core. The theoretical calculations indicate that the data transmission bus bandwidth can reach 4 Gbps, while in the meantime its reliability has been proved by experiments. The experimental results show that the system owns the characteristics of high data sampling accuracy, stable and reliable high-speed transmission, and has promising application prospects.
\end{abstract}

Keywords: seafloor earthquake observatories; Zynq-7000 Soc; clock synchronization; transmission system

\section{Introduction}

As the largest area on the earth, the ocean is of great significance for regulating the global environment and climate, maintaining the global ecosystem. Abundant natural resources and economic benefits are obtained by reasonable exploration and utilization of the ocean $[1,2]$. However, frequent marine disasters cause a considerable threat to human life and property in individual productive marine activities. Therefore, the forecast of marine disasters has become a key issue in marine research. On this basis, researchers pointed out that the establishment of seafloor observatories could solve this problem effectively [3]. Seafloor observatories can detect the ocean internal environment stably during a long period and provide long-term, real-time submarine data. With the aid of the inversion of the sampled data, the early warning capabilities of marine disasters can be highly improved.

More than 85 percent natural earthquakes take place in the ocean; however, their monitoring have been still relatively weak. Nowadays, natural submarine earthquakes observation, based on the seafloor observatories, has become a novelty type of observed method. 
In the international, it is represented by American-MARS [4], Canadian-NEPTURE [5], Japanese-DONET [6] and EU-EMSO [7]. As for in China, the research team of Tongji University has done a lot of work in this field and put forward many innovative design schemes in the East China Sea [8-10]. From the operation of the seafloor observatories, we can recognize that seabed seismic observatories can be more effectively for monitoring small earthquakes that occur on the seabed and provide early warning for marine disasters [11]. To summarize, in addition to Japan's seafloor observatories, others are still single-point, seafloor surface monitoring. The seismograph and the junction station can only be within a short distance $(50 \mathrm{~m})$, which cannot meet the requirements for networked monitoring of submarine earthquakes [12-14].

With continuous investigation on seafloor observatories, some novelty or improved seafloor earthquake observed schemes have been proposed. Yu et al. first proposed an object model for regulating the sensor control and data sampling of seafloor observatories, which is conceptually designed as a group of sensor resource objects. Based on the properties and operations of these objects, the client-server sensor control architecture can realize bidirectional information flow of control commands and sampled data [8]. Zhan et al. successfully sensed earthquake waves and water waves over a 10,000-km submarine cable connecting Los Angeles, California and Valparaiso, Chile by monitoring the polarization of conventional optical communication channels. Without special equipment, laser sources or dedicated fibers requirements, their method exhibits very convenient scalability to convert global submarine cables into continuous real-time seismic and tsunami observatories [15]. Diouf et al. created a PoF and communication system for extending seafloor observatories, which is based on an optical structure to simultaneously transmit power and data through the same single-mode fiber. Raman scattering effect is used to amplify the optical data signal, reducing the power consumption of sensor nodes [16]. Bruce et al. discussed the blueprint of intelligent submarine cable system, which integrated sensors into the future submarine telecommunication cables. The simulation results showed that cable-based pressure and seismic acceleration sensors could effectively improve the tsunami warning time and seismic parameters [17].

In this paper, we investigate a chained data sampling and transmission system (SQSTS), based on Zynq-7000 Soc and clock synchronization, which can be used as a prototype and foundation for a new seafloor observatory. Compared with conventional sensor web prototype [9], SQSTS replaced the traditional undersea junction box with the control module based of Zynq-7000 Soc. In this way, we can keep the overall architecture of the usual seafloor observatory system and expand its functions. Besides that, we also introduce the clock synchronization module to ensure that the time stamp in each seismometer node can reach the $\mu$ s level, laying a solid foundation for supporting subsequent seismic data inversion operations. Compared with the ocean bottom cabled seismometer system, which is similar to our system in structure, the main control module of SQSTS uses the Zynq7000 Soc with both FPGA (PL side) and ARM (PS side) [11]. In consequence, an additional CPU is not demanded to control data acquisition and data transmission functions. The transmission between nodes of SQSTS uses the gigabit transceiver in the physical layer of the Zynq7000 Soc. Compared with Ethernet transmission (100 Mbps), it can achieve $4 \mathrm{Gbps}$ high-speed data transmission. Furthermore, in contrast to the previous clock synchronization system with a time stamp accuracy of $0.1 \mathrm{~ms}$, SQSTS adopts a new method based on the PPS signal and the internal clock cycle of the FPGA to extend the minimum time unit of the time stamp to the $\mu$ s level and the theoretical accuracy is $1 \mu \mathrm{s}$.

The content of our article is organized as follows: In Section 1, the expected goals that SQSTS needs to achieve, and the overall system design scheme are introduced. In Section 2, the classification of nodes in SQSTS and the selection of hardware modules in nodes are proposed. In Section 3, the software functions to be realized by each node in SQSTS and their designs are shown. In Section 4, the SQSTS model and experimental results, as well as a discussion of the results, are presented. 


\section{Materials and Methods}

\subsection{System Overall Scheme Design}

As the prototype of the seafloor observatories, the SQSTS designed in this paper have the basic function of the seafloor observatories: data acquisition. Thus, a data acquisition module is indispensable in our system. Considering that SQSTS is mainly used for the observation and early warning of submarine earthquakes, the submarine data in this article therefore specifically refers to submarine seismic signals. In addition, in order to support the subsequent processing and analysis of the sampled data, it is also necessary to add a clock synchronization module to the SQSTS to provide accurate time stamps for the data sampled at each moment. Finally, a specific control module is also required, which is similar to a traditional junction box to realize functions such as controlling data collection and transmission. The specific design of each module is as follows.

With the increase of the number of submarine sensors and sensor signal channels, the high-speed data transmission of the link is much more desirable than before. In order to realize high-speed data transmission between nodes of submarine optical cable link, SerDes (SERializer/DESerializer) communication technology is adopted in this paper. SerDes is a point-to-point, time-division multiplexed transmission technology, which can greatly decrease the number of channels and chip pins required for data transmission thus, reduce the cost of data transmission. As an important serial communication bus, SerDes offers advantages of low power consumption, strong anti-interference ability, fast speed, long transmission distance, etc. Hence, it is very suitable for application in SQSTS.

For the control module of each node in the SQSTS, it is inevitable to own the ability of controlling multiple secondary devices at the same time, facilitating data reading and fusion processing, and interacting with the other main control chips of the last/next level nodes by using SerDes communication technology. Considering the massive application in the future networking, the cost should be minimized as much as possible on the premise of satisfying the chip function and reliability.

In order to meet the requirements of the high-precision seismic signals sampling and have the ability to communicate with the main control chip in full duplex working mode, we intend to adopt an AD sampling module with an accuracy of up to 24-bit, high sampling rate, and supporting for SPI protocol. Moreover, in the clock synchronization module, the clock synchronization accuracy is demanded to reach ns level, and the time stamp is required to reach $\mu$ s level.

\subsection{System Hardware Design}

\subsubsection{Overall Design}

SQSTS is composed of three types of seismometer nodes, which are classified as the front node, the intermediate node and the end node according to their distribution locations. The components of the hardware circuit of each node are divided into three parts: the control part (primary device), the data sampling part (secondary device), and the clock synchronization part (secondary device). The components of each part are shown in Table 1.

Table 1. Device composition table of different components in the node.

\begin{tabular}{ccc}
\hline Control Part & Data Sampling Part & Clock Synchronization Part \\
\hline & & Master Clock: EGM Multi-function \\
Zynq-7000 Soc Main & ADS1256 Data Sampling & Clock Synchronization Master Clock \\
Control Board & Card & Slave Clock: P88 1588 PTP Clock \\
& & Synchronization Board \\
\hline
\end{tabular}

In each node, the control part is responsible for receiving and fusing the data uploaded by each secondary device; the data sampling part is responsible for sampling submarine data; the clock synchronization part is responsible for synchronizing with the master clock and providing time stamp. The nodes are connected by optical cables to form a 
transmission link. Each node packs its own data and data from the last level node, and then sends them to the next level node. Finally, the end node sends all nodes' data, the link data to the data center through the Gigabit Ethernet. The whole system block diagram is shown in Figure 1.



Figure 1. System block diagram of SQSTS.

\subsubsection{Component Design}

Considering the design requirements of the main control module of each node, the zynq7000 Soc developed by Xilinx company, which adopts the ARM Cortex A9 architecture, is chosen as the main control chip. The chip has PL side (FPGA) and PS side (ARM) and supports multiple secondary interfaces to meet the interface requirements of the peripherals used in each node. PL side means the side of programmable logic, which contains a Field Programmable Gate Array (FPGA) and owns parallel processing capability. PS side means the side of processing system, which contains an ARM cortex- A9 processor, and owns serial processing capability. Apart from that, the Gigabit Transceivers (GTX) and Ethernet PHY chips are used in the system.

ADS1256 data sampling chip produced by TI Company is selected for data sampling. The accuracy of the chip can reach 24 bits, and the chip has 8 channels with the sampling rate of 30KSPS, which satisfies the performance requirements of the data sampling part of our system. The external $5 \mathrm{~V}$ input required by the chip can be directly provided by the control part, and the SPI bus is connected with the MIO ports on the PL side of zynq-7000 SOC.

In order to ensure that the time information of each node is synchronized so as to obtain accurate time stamp, a clock synchronization system needs to be added to each node; thus, the P88 1588 PTP clock synchronization board (Produced by Coolshark Company) is selected as the slave clock in the node. The board can be connected to the master clock through an optical cable, keeping the time consistent with the master clock. Meanwhile, the board can be connected to Zynq-7000 Soc through the GPIO port to provide synchronized time information (in PPS and ToD formats). The EGM master clock (Produced by Coolshark Company, Beijing, China) is selected as the master clock, whose clock source is satellite 
(GPS, Beidou, Glonass). The output form can be set up as IEEE1588 PTP, SyncE and time signals (frequency, PPS, ToD) through optical ports. It has a distributed synchronous design architecture, which can expand the number of unicast slaves up to 450 . It features all the characteristics of the IEEE1588 master clock and boundary clock, also achieves effective management and maintenance, and provides a continuous and accurate time information after the loss of the relevant reference source signal.

\subsection{System Software Design}

\subsubsection{Software Architecture Design}

Considering the functional distinctions caused by the different distribution positions of the nodes, it is necessary to divide the nodes into three categories: front node, intermediate node and end node, and write corresponding Verilog HDL programs according to the functions of each class. For the front node, the functions that need to be achieved include the controlling and reading the data sampling part and the clock synchronization part, as well as the fusing and packaging of time, MAC address, sampled data and other data, and finally sending it to the next level node through the optical cable. For the intermediate node, besides the whole functions of the front node, it also needs to have the functions of receiving data from the last level node and merging and repackaging with its own data. Finally, for the end node, not only all functions of intermediate node except the function of sending data to the next level node are required, but also the PS side of the end node needs to be used to send the data of the entire link to the data center through Gigabit Ethernet. The functions of different types of nodes are shown in Figure 2.

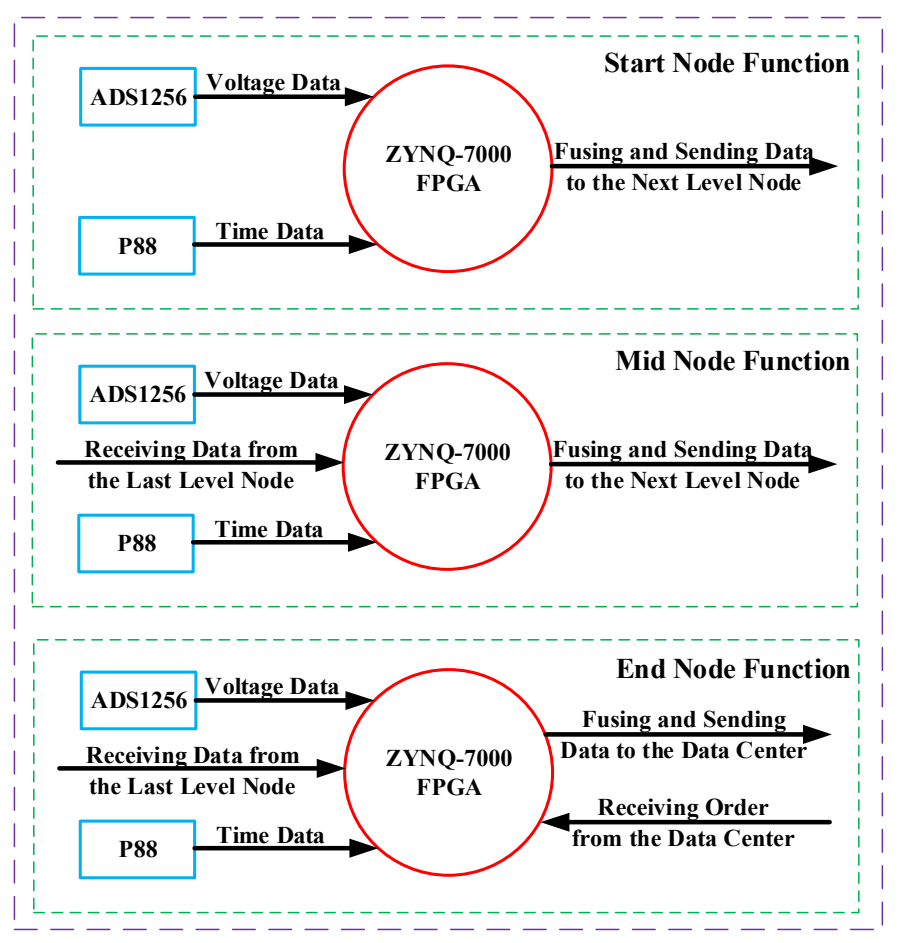

Figure 2. Function diagram of different types of nodes.

\subsubsection{Data Sampling Module Design}

Zynq-7000 Soc reads the sampled data in ADS1256 through the SPI protocol. The specific process is as follows: When Zynq-7000 Soc needs to communicate with ADS1256, it pulls down the chip selection signal-CS, and uses the clock signal SCLK to control the data reading and writing of ADS1256. The MOSI signal is responsible for configuring the state of the registers in the ADS1256. When detecting that the DRDY signal is set in the high voltage level, the Zynq-7000 Soc can obtain the sampled data of the ADS1256 from the MISO signal. 


\subsubsection{Clock Synchronization Module Design}

The P88 1588 PTP clock synchronization board transmits the ToD information which is in the NMEA ZDA format and PPS information to the Zynq-7000 Soc. The Zynq-7000 Soc can use the ToD information to obtain a time stamp of the second level. However, the requirements of our system are time stamps up to the microsecond level. Thus, we designed a program to obtain microsecond level time information by using the PPS signal provided by the P88 1588 PTP clock synchronization board and the inner crystal oscillator of the Zynq-7000 Soc. The specific process is as follows: Set the period of the PPS signal to $1 \mathrm{~s}$ with the pulse width of $300 \mu \mathrm{s}$. The crystal oscillator used on the PL side of the Zynq-7000 Soc is $100 \mathrm{MHz}$, which means that its clock cycle is $10 \mathrm{~ns}$. When the rising edge of each clock cycle arrives, the Zynq-7000 Soc reads the PPS signal. If the PPS signal is set in the low voltage level at this time, it starts to count the clock cycle. When the Zynq-7000 Soc detects that the PPS signal is set in the high voltage level, the counter returns to zero and stops counting. It can be seen that this method does not need to add any other peripherals and makes full use of the resources inside the node. It is simple and reliable, and its theoretical error accuracy is $\pm 1 \mu \mathrm{s}$.

The equation can be used to obtain microsecond level time information:

$$
\text { Time_ } \mu \text { s }=(\text { Count of Clock Cycle }+10) / 100+300
$$

The flow chart of the control of clock synchronization module is shown in Figure 3.



Figure 3. The flow chart of the control of clock synchronization module.

\subsubsection{Design of Transmission Link Module}

In the optical cable transmission design between nodes, we used the Aurora IP core, which was embedded in the Vivado compilation environment by Xilinx Company. The IP core can conveniently call the physical layer device-Gigabit transceiver for optical cable data transmission. The schematic diagram and design configuration parameters of the IP core are shown in Figure 4. 


\begin{tabular}{l|l|} 
Physical Layer & \multicolumn{2}{c}{} \\
Lane Width (Bytes) & 4 \\
Line Rate (Gbps) & 6.25 \\
GT Refclk (MHz) & 125.000 \\
INIT clk (MHz) & 100 \\
DRP Clk (in MHz) & 100 \\
\end{tabular}

(a)

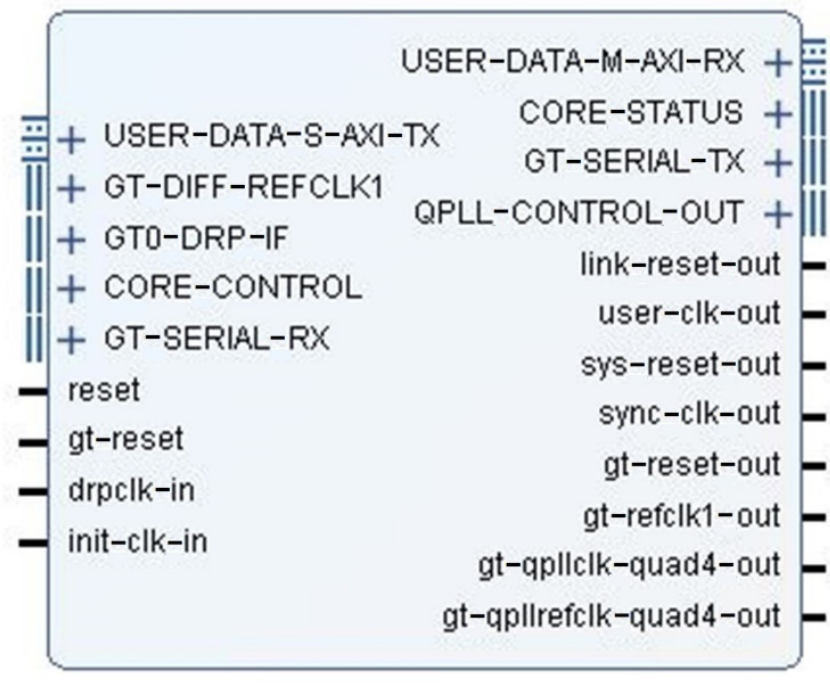

(b)

Figure 4. (a) Aurora IP core design configuration parameters; (b) Aurora IP core schematic diagram.

From the figure, it can be seen that the maximum bandwidth is set to $6.5 \mathrm{Gbps}$, the data length of each transmission is 4 bytes, and the reference clock is $125 \mathrm{MHz}$. The link bus bandwidth can be $4 \mathrm{Gbps}$ according to the following calculation equation:

$$
\text { Band Width }=\text { Clock Frequency } * \text { Bits }
$$

In the procedure of data transmission between nodes, we use the similar Aurora IP core, and the design parameters, working mode and other configurations. As a result, a unified design of the IP core can be customized. However, we must consider the functional distinctions caused by the different locations of each node, which further leads to differences in the timing design of various types of nodes. The specific timing design of this paper are as follows: In the timing design of the transmission program of the front node, only the function of sending data packets to the next level node needs to be operated. Therefore, it can be sent as soon as the data packet is packaged. In the timing design of the transmission program of the intermediate node, not only the function of sending data packets to the next level node, but also the function of receiving data packets from the last level node must be deliberated. Hence, in the design, the data packet sent by the last level node must be received first, and then the received data from the last level node and the data of this level node are packaged and sent. In the timing design of the transmission program of the end node, it is necessary to consider not only its function of receiving data packets from the last level node, but also its function of sending data packets to the data center. Consequently, in the design, the data packet sent by the last level must be received first, and then the received data from the last level node and the data of this level node can be packaged and sent to the data center.

The composition sequence of the data packet between nodes is Header, Seismometer number, Time stamp, Sampling data, MAC address, Separator, and its format is defined as follows in Table 2. 
Table 2. Data packet format transmitted between nodes.

\begin{tabular}{cccc}
\hline Function & Form & Bytes & Example \\
\hline Header & $0 \mathrm{x}$ & 4 & AA 55 AA 55 \\
Seismometer number & $0 \mathrm{x}$ & 1 & 01 \\
Time stamp & ASCII & 17 & 3132303234303431303630393038037 C 51 \\
Sampling data & $0 \mathrm{x}$ & 3 & 56 A0 09 \\
MAC address & $0 \mathrm{x}$ & 3 & 000000000001 \\
Separator & $0 \mathrm{x}$ & 1 & FF \\
\hline
\end{tabular}

This section may be divided by subheadings. It should provide a concise and precise description of the experimental results, their interpretation, and the experimental conclusions that can be drawn.

In our design, the end node of the transmission link needs to merge and package the data of all nodes and send it to the data center. Taking into account the practicality of implementation and security of the transmission form, as well as the scalability of the transmission data width, Gigabit Ethernet is adopted as the information transmission method between the end node and the data center. This method takes advantage of both the PL side and the PS side of the Zynq-7000 Soc at the same time: the data fusion and package on the PL side can fully utilize the efficient parallel processing capabilities of the PL side. Data transmission is carried out on the PS side, which can take full advantage of the soft-core architecture on the PS side and program to realize Gigabit Ethernet transmission easily.

Xilinx company provides LwIP function support package in the development environment of PS side. By using it, the Gigabit Ethernet transmission based on the TCP server protocol can be quickly realized. PL side and PS side interact with each other through AXI bus. PL side transmits link data to PS side through AXI bus, and the PS side can transmit the user's control commands for link data transmission to the PL side through AXI bus: transmission start, stop and reset too. We set the PS side to send a data packet to the data center every $10 \mathrm{~ms}$.

The data packet transmitted between the PS side and the data center is composed of Header, Packet Index, Length, Data (combined end node data, intermediate node data with front node data), and its format is defined as shown in Table 3.

Table 3. Data packet format transmitted between the PS side and the data center.

\begin{tabular}{cccc}
\hline Function & Form & Bytes & Example \\
\hline Header & $0 \mathrm{x}$ & 24 & AA 55 AA 55 AA 55 AA 55 AA 55 AA 55 \\
$\begin{array}{c}\text { Packet Index } \\
\text { Length }\end{array}$ & ASCII & 4 & AA 55 AA 55 AA 55 AA 55 AA 55 AA 55 \\
& $0 \mathrm{x}$ & 4 & 00 00 0D E4 \\
Data & $0 \mathrm{n}$ & 84 & 00000054 \\
& & & $013132303234303431383039303630037 \mathrm{C} 5100000000000156 \mathrm{A0} 09 \mathrm{FF} 023132$ \\
& & & $303431383039303630037 \mathrm{~B} 5900000000000329316 \mathrm{FFF}$ \\
\hline
\end{tabular}

\section{Results}

\subsection{Experiment}

The completed SQSTS model is shown in Figure 5. Node-1 represents the end node, Node- 2 represents the intermediate node, and Node- 3 represents the front node. The PC simulates a data center, and a sliding rheostat simulates the sampled signal source. Each node includes a Zynq-7000 Soc main control board, an ADS1256 sampling card and a P88 1588 PTP clock synchronization board. The nodes are connected by optical cables to form a transmission link. The P88 1588 PTP clock synchronization board of each node and the EGM master clock are also connected by optical cables to form a master-slave clock synchronization network to provide synchronized time stamp. Node-1 finally sends the 
link data to the PC via Gigabit Ethernet. Through the host computer program on the PC, the received link data can be read out.

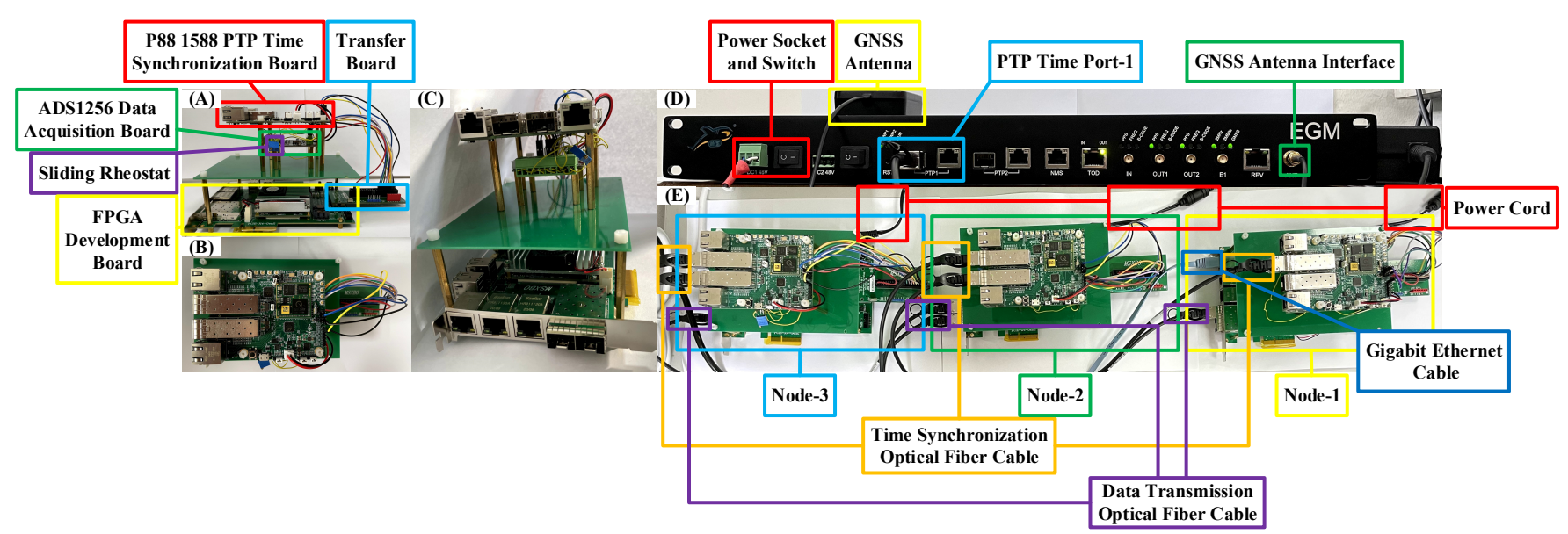

Figure 5. (A) Node main view and its components; (B) Node top view; (C) Node side view; (D) EGM master clock photo and interface description; (E) Transmission link photo and wiring relationship between nodes.

\subsection{Result Analysis}

\subsubsection{The Verification of Link Transmission Function}

The link data read by the PC is shown in Figure 6, and the results obtained are analyzed below. The content of a link data packet displays in the red box. According to the design, the first is the frame header of the data packet, which corresponds to the yellow box. The following is the number of the data packet, which corresponds to the orange box. And next is the length of the data packet, which corresponds to the dark blue box. Then, the data of the front node corresponds to the blue box, the data of the intermediate node corresponds to the green box, and the data of the end node corresponds to the purple box. It can be seen that the data format of each node is Header, Seismometer number, Time stamp, Sampling data, MAC address and Separator, which is consistent with the design, indicating correctness of the link data transmission. By calculating the time difference between every two packets of the same node, it can be found that the value is $10 \mathrm{~ms}$, which is consistent with expected design: the end node PS side sends a data packet to the data center every $10 \mathrm{~ms}$.

\subsubsection{The Verification of Clock Synchronization}

From the data packets which are sent from the end node to the PC end, we can obtain the TOD information of the three nodes. After checking an amount of data packets, we found that the time information of the nodes: year, month, day, hour, minute, and second are the same, which can demonstrate the synchronization of the ToD information. Besides that, we also read and display PPS signals provided by P88 1588 PTP clock synchronization board of each three nodes and measure the PPS signals' high level width by using Tektronix oscilloscope to verify the synchronization accuracy of these signals. The digital waves displayed by the oscilloscope are shown in the Figure 7. Considering that the level standard of PPS signal is TTL standard, the high level of each channel is set to $2.40 \mathrm{~V}$. In a large range (the scale of oscilloscope is $200 \mu \mathrm{s}$ ), it can be observed that the high-level width of PPS signals of three nodes are $300 \mu \mathrm{s}$, which is consistent with the duration of high-level pulse of $300 \mu \mathrm{s}$ in our design, and posedges and negedges of the three PPS signals are also keep the synchronous changes. When observing in a small range (the scale of oscilloscope is $1 \mu \mathrm{s})$, we can see the posedges of PPS signals of three nodes more meticulously, and the signals still keep a precise synchronization. Thus, we can draw the conclusion that the PPS signals of the three nodes are synchronized. As for the initial clock signals of the three nodes, we do not use a specific method to keep them in synchronization like PPS signals. 
However, because the maximum difference between random two clock signals of the three is half of their period: $5 \mathrm{~ns}$, it cannot have a significant effect on our time synchronization precision. In the Zynq-7000 Soc programming language, when getting a reminder in the division calculation, the result will be rounded automatically. Thus, using division in our program can produce an error accuracy of $\pm 1 \mu \mathrm{s}$. In conclusion, we have verified that we can calculate the time of $\mu$ s level by using PPS and clock signals, and the precision of our method is $\pm 1 \mu \mathrm{s}$. For future practical seafloor observatory applications, the distance between nodes is usually more than ten kilometers, so the time delay caused by PPS signal transmission in optical cable must be taken into account. We can estimate the time by using the transmission speed of electromagnetic wave in copper cable and the distance between nodes and configure on P88 1588 PTP clock synchronization board in advance to subtract the transmission time for compensating.

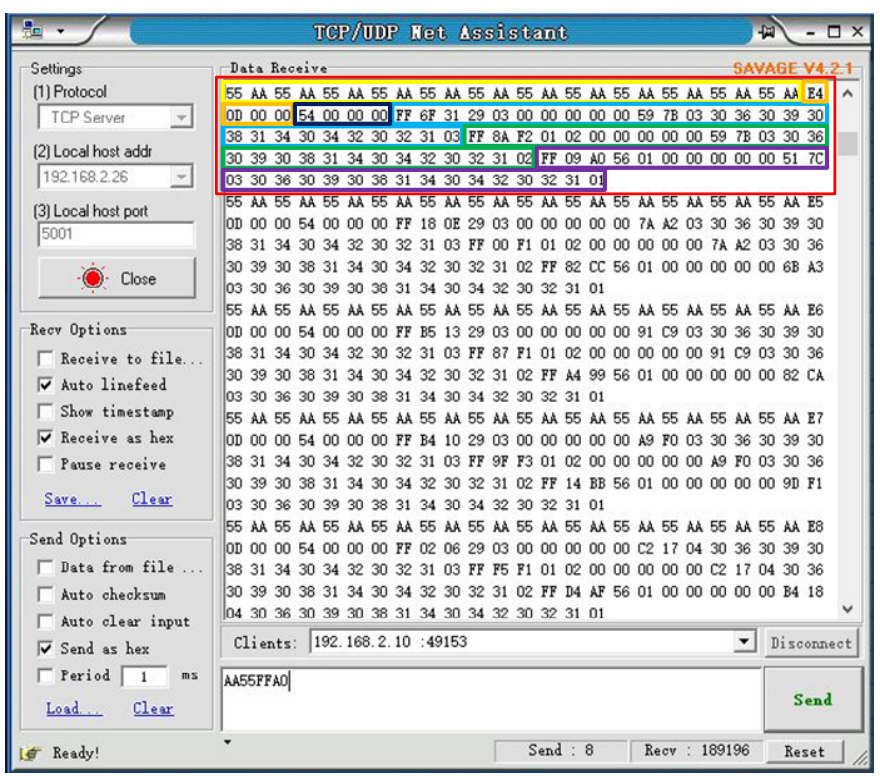

Figure 6. Link data read by the host computer.

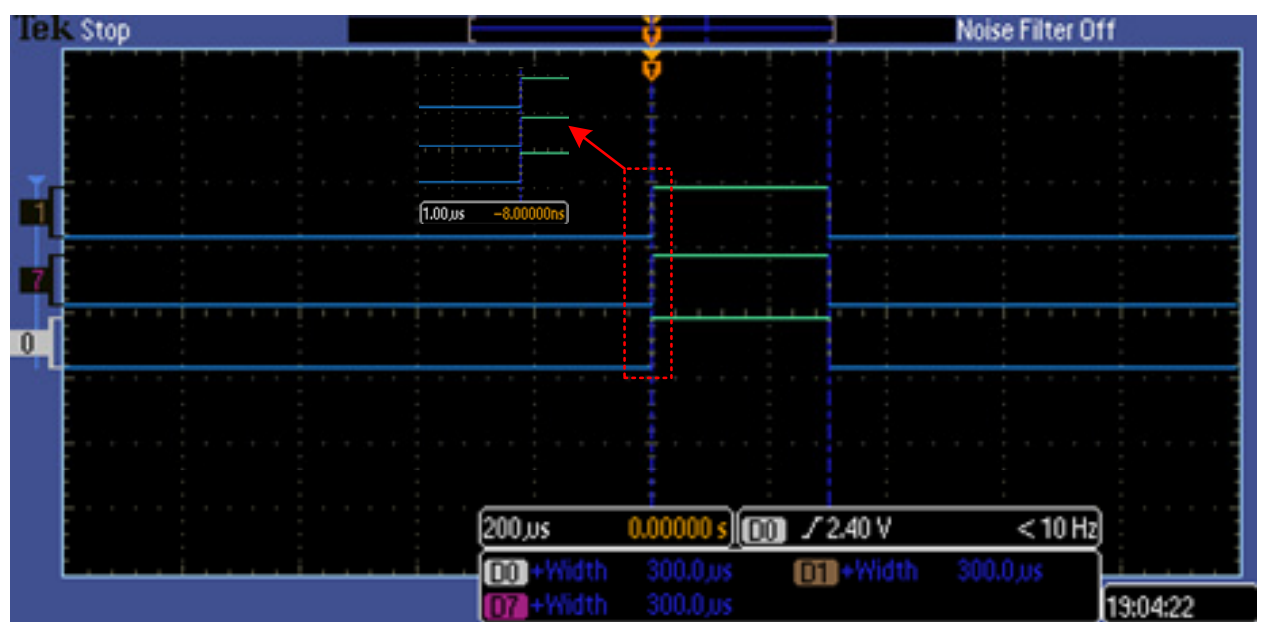

Figure 7. PPS signals read by oscilloscope.

\subsubsection{Summary}

The above-mentioned experimental phenomena show that the data transmission link has realized the design functions, and also verify the correctness and stability of the clock synchronization system. After the system has been running for a long time, no packet loss has been observed, proving the stability and reliability of the data transmission system. 
It is worth noting that the phenomenon of data inversion appears, because the highorder byte is sent first and then the low-order byte during an Ethernet transmission. Using the network device manager on the PC, the transmission speed of Ethernet has reached the standard of Gigabit Ethernet.

\section{Discussion}

This article describes a chained data acquisition and transmission system (SQSTS), which can be used as the basic component of the cabled seafloor earthquake observatories. Based on the Zynq-7000 Soc, the control module can replace the traditional junction box and realize the functions of controlling high-precision data acquisition and high-speed transmission (4 Gbps). On the basis of the clock synchronization technology, it is possible to provide a time stamp up to the microsecond level for the sampled data, supporting for the subsequent seismic data inversion operation and earthquake early warning. The system has provided satisfactory actual performance through a series of tests and applications. We have proved by this research that the design and implementation of SQSTS is beneficial to the research of seafloor observatories. In the next step, we will develop a truly practical, cabled seafloor earthquake observatory based on SQSTS.

Author Contributions: Conceptualization, W.L., J.L. and J.Z.; methodology, W.L. and J.Q.; development and validation, J.Q., W.L.; writing-original draft, J.Q. and W.L.; writing-review and editing, J.Q. and W.L. All authors have read and agreed to the published version of the manuscript.

Funding: This research was funded by the Oceanic Interdisciplinary Program of Shanghai Jiao Tong University (SL2020ZD205), Scientific Research Fund of Second Institute of Oceanography, MNR (SL2020ZD205) and National Natural Science Foundation of China (No. 61871266).

Institutional Review Board Statement: Not applicable.

Informed Consent Statement: Not applicable.

Data Availability Statement: Not applicable.

Conflicts of Interest: The authors declare no conflict of interest.

\section{References}

1. Aguzzi, J.; Chatzievangelou, D.; Marini, S.; Fanelli, E.; Danovaro, R.; Flögel, S.; Lebris, N.; Juanes, F.; De, L.F.C.; Rio, J.D.; et al. New High-Tech Flexible Networks for the Monitoring of Deep-Sea Ecosystems. Environ. Sci. Technol. 2019, 53, 6616-6631. [CrossRef] [PubMed]

2. Aguzzi, J.; Chatzievangelou, D.; Francescangeli, M.; Marini, S.; Bonofiglio, F.; Rio, J.D.; Danovaro, R. The Hierarchic Treatment of Marine Ecological Information from Spatial Networks of Benthic Platforms. Sensors 2020, 20, 1751. [CrossRef] [PubMed]

3. Buck, J.J.H.; Bainbridge, S.J.; Burger, E.F.; Kraberg, A.C.; Casari, M.; Casey, K.S.; Darroch, L.; Del-Rio, J.; Metfies, K.; Delory, E.; et al. Ocean Data Product Integration Through Innovation-The Next Level of Data Interoperability. Front. Mar. Sci. $2019,6,32$. [CrossRef]

4. Massion, G.; Raybould, K. MARS: The monterey accelerated research system. Sea Technol. 2006, 47, 39-42. [CrossRef]

5. Barnes, C.R.; Best, M.M.R.; Johnson, F.R.; Pautet, L.; Pirenne, B. Challenges, Benefits, and Opportunities in Installing and Operating Cabled Ocean Observatories: Perspectives From NEPTUNE Canada. IEEE J. Ocean. Eng. 2013, 38, 144-157. [CrossRef]

6. Mulia, I.E.; Satake, K. Developments of Tsunami Observing Systems in Japan. Front. Earth Sci. 2020, 8, 145. [CrossRef]

7. Lefevre, D.; Zakardkjian, B.; Embarcio, D. Unique observatories for sea science and particle astrophysics: The EMSO-Antares and EMSO-Western Ionian nodes in the Mediterranean Sea. Eur. Phys. J. Conf. 2019, 207, 09004. [CrossRef]

8. Yu, Y.; Xu, H.; Xu, C. An Object Model for Seafloor Observatory Sensor Control in the East China Sea. J. Mar. Sci. Eng. 2020, 8, 716. [CrossRef]

9. Yu, Y.; Xu, H.; Xu, C. A Sensor Web Prototype for Cabled Seafloor Observatories in the East China Sea. J. Mar. Sci. Eng. 2019, 7, 414. [CrossRef]

10. Yu, Y.; Xu, H.; Xu, C. A Sensor Control Model for Cabled Seafloor Observatories in the East China Sea. Sensors 2018, $18,3027$. [CrossRef] [PubMed]

11. Shinohara, M.; Kanazawa, T.; Yamada, T.; Machida, Y.; Sakai, S. New compact ocean bottom cabled seismometer system deployed in the Japan Sea. Mar. Geophys. Res. 2014, 35, 231-242. [CrossRef]

12. Peng, X.T.; Zhou, H.Y.; Wu, B.C.; Lu, F.; Wu, Z.W.; Yang, C.J.; Li, P.L.; Li, D.J.; Jin, B.; Feng, Z.P.; et al. Test China Node on Monterey Accelerated Research System(MARS). Adv. Earth Sci. 2011, 26, 991-996. [CrossRef] 
13. Yang, C.J.; Zhang, F.; Chen, Y.H.; Li, D.J.; Jin, B. Technologies of junction box for seafloor observation network. J. Mech. Eng. 2015, 51, 172-179. [CrossRef]

14. Hao, T.Y.; Guo, Y.G.; Zhang, Y.F.; You, Q.Y.; Xie, Z.Z.; Liu, S.H.; Yang, J.; Xu, X.Q.; Zhou, L.J.; Zheng, S.P.; et al. Cabled seafloor observation technology: A case study of the submarine seismic station in Wuyu Island. Chin. J. Geophys. Chin. Ed. 2019, 62, 4323-4338. [CrossRef]

15. Zhan, Z.; Cantono, M.; Kamalov, V.; Mecozzi, A.; Müller, R.; Yin, S.; Castellanos, J.C. Optical polarization-based seismic and water wave sensing on transoceanic cables. Science 2021, 371, 931-936. [CrossRef] [PubMed]

16. Diouf, C.; Quintard, V.; Ghisa, L.; Guegan, M.; Perennou, A.; Gautier, L.; Tardivel, M.; Barbot, S.; Dutreuil, V.; Colas, F. Design, Characterization and Test of a Versatile Single-Mode Power-Over-Fiber and Communication System for Seafloor Observatories. IEEE J. Ocean. Eng. 2020, 45, 656-664. [CrossRef]

17. Howe, B.M.; Arbic, B.K.; Aucan, J.; Barnes, C.R.; Weinstein, S. SMART Cables for Observing the Global Ocean: Science and Implementation. Front. Mar. Sci. 2019, 6, 424. [CrossRef] 